\title{
Determination of the coefficient of expansion of a carbon tube and its assembly for thermal compensation of metrological structures
}

\author{
Salma EL ASMAI ${ }^{1}{ }^{*}$, François HENNEBELLE ${ }^{1}$, Thierry COOREVITS ${ }^{2}$, Jean-François \\ FONTAINE $^{1}$ \\ ${ }^{1}$ UBFC, Avenue des plaines de l'Yonne, 89000 Auxerre, France \\ ${ }^{2}$ Ecole nationale supérieure des Arts et Métiers - Laboratoire MSMP, Lille, France
}

\begin{abstract}
Composite materials are increasingly used in 3D metrology devices. Their use is justified by their interesting mechanical properties including their low density and good rigidity but especially their low coefficient of thermal expansion. In fact, in order to improve production efficiency, companies nowadays integrate more and more control equipment directly in situ. These are then subject to thermal variations. The use of composite materials is an interesting approach. However, in some cases, the lack of knowledge of their coefficient of thermal expansion and their behavior might increase measurement uncertainties. The objective is to study the thermal behavior of a carbon tube alone and the same tube with aluminium fixing elements at its extremities, in order to determine the coefficients of expansion of the carbon alone and to quantify the influence of the fixation with aluminium elements. This experiment makes it possible to directly compensate the dimensional variations of the metrological structure depending on the temperature variations and thus to limit measurement uncertainties. The thermal expansion coefficients of the carbon tube and its assembly are determined by measuring relative variations in height with a ZERODUR ${ }^{\circledR}$ reference bar. The whole is positioned in a climatic chamber. ${ }^{*}$
\end{abstract}

\section{Introduction}

Tubes made of braided carbon fiber are increasingly used in metrological structures. Indeed, in addition to their lightweight, their coefficient of thermal expansion (CTE) is almost zero. In the context of the factory of the future 4.0 and the installation of metrology equipment closer to production, these products are very interesting and even unavoidable. As a result, segmented Ball Bars or Articulated Arm Coordinate Measuring Machines (AACMM) for example use this type of components in their structure in order to guarantee the dimensional stability of the equipment and thus the traceability of the measurements during thermal variations.

\footnotetext{
*Corresponding author: salma.el-asmai@u-bourgogne.fr
} 
Carbon tube manufacturers traditionally indicate on their product records a coefficient of thermal expansion equal to zero for these carbon fiber bars with an associated uncertainty of $2.10^{-6} \mathrm{~K}^{-1}$ or they provide only the coefficient of the fiber regardless of the matrix and fiber orientation. Thus, the experiments carried out to determine the CTE of carbon fiber tubes mainly address the case where the tubes are subject to significant temperature variations (from $-60{ }^{\circ} \mathrm{C}$ to $180^{\circ} \mathrm{C}$ ). In these cases, systems with a "medium" measurement uncertainty can be used, namely Linear Variable Differential Transformer (LVDT) sensors [1], optical systems based on interferometry [2] and so on. Note that today, it is possible to design composite materials based on carbon nanotubes with a coefficient of negative thermal expansion [3].

The objective of the work is to estimate the real expansion coefficient of these tubes, in particular given that they are assembled on structures via two aluminium layers, one at each end of the tube. To do this, we will determine two different expansion coefficients, namely the CTE of the carbon fiber tube alone and the CTE of the assembly made of the carbon tube and the aluminium alloy.

It will be demonstrated that the CTE of an assembled carbon tube is therefore not necessarily negligible in terms of measurement uncertainties, when the measuring devices are used in the shop of floor and industrial environments where the temperature is not regulated. This problem is at the heart of the constraints of the factory of the future.

\section{Justification of the testing method}

To determine The CTE of a braided carbon tube (of annular section) with low uncertainty is not easy.

Therefore, it is impossible to perform measurements by extensometry to determine the coefficient of expansion of this type of structure. In fact, since the CTE is small, if we consider that the CTE of the carbon fiber in the composite matrix has is $10^{-6} \mathrm{~K}^{-1}$, then the total elongation of a tube $500 \mathrm{~mm}$ long is $0.5 \mu \mathrm{mK}^{-1}$. In order to carry out this experiment, it is necessary to heat the tube. This is problematic because the order of magnitude of measurement uncertainties of the gauges under the influence of temperature variations is the same as the dimensional variation that can be observed.

It is not conceivable either to directly measure the variation of the tube length under the effect of temperature variations thereof, even on a good three-dimensional measuring machine (CMM). Indeed, the measurement uncertainties of the CMMs being a function of the measured length, the measurement uncertainty induced over a length of $500 \mathrm{~mm}$ will be close to $1 \mu \mathrm{m}$, without considering the disturbances of the machine under the effect of the temperature of the tube.

To achieve this type of measurement, one of the solutions is to measure by "length comparison" by means of another reference tube whose characteristics are perfectly known. The choice was to use a ZERODUR ${ }^{\circ}$ bar with a very low CTE $\left(10^{-8} \mathrm{~K}^{-1}\right)$ [4] of the same nominal length as the tube to be characterized, and to measure the relative length variation between the ZERODUR $\AA$ and the tube at different temperatures. Knowing the temperature and the variation of the relative length, it is easy to evaluate the CTE of the tube.

\section{Set up of the experiment and the heating section}

The objective is to create a temperature-controlled heating section that can be used to test braided composite tubes. The upper face of the ZERODUR ${ }^{\circledR}$ and the upper plane of the tube must be accessible to be measured with a touch probe by the CMM. The heating section must 
be insulated to allow stabilization and regulation of the heating section but also to avoid heating the machine locally, which would lead to measurement errors.

The CMM used is the machine ZEISS UPMC 8/12/6 Arts et Métiers ParisTech, center of Lille, equipped with a measuring head VAST Gold type. The measurement uncertainty in the measurement direction ( $\mathrm{Z}$ axis) of the $\mathrm{CMM}$ is $0,5 \mu \mathrm{m}+\frac{L[\mathrm{~mm}]}{900} \mu \mathrm{m}(\mathrm{k}=2)$.

The ZERODUR ${ }^{\circledR}$ bar was custom-made by SCHOTT [5], has a length of $510.035 \mathrm{~mm}$ and a diameter of $30 \mathrm{~mm}$. It is held on a specific support allowing it to be held unrestrained in a vertical position (Fig. 1a). The heating system is provided by a heating tube made by the composite team of Arts et Métiers ParisTech, Center of Lille. A heated wired network sewn before polymerization on a composite web is arranged around a zinc tube. A specific box makes it possible to regulate at a defined setpoint temperature. The temperature is measured in two points of the carbon tube through two probes PT100 son 4 disposed on small copper plates for physical filtering thermal inertia of the temperature measurement (Fig. 1b).
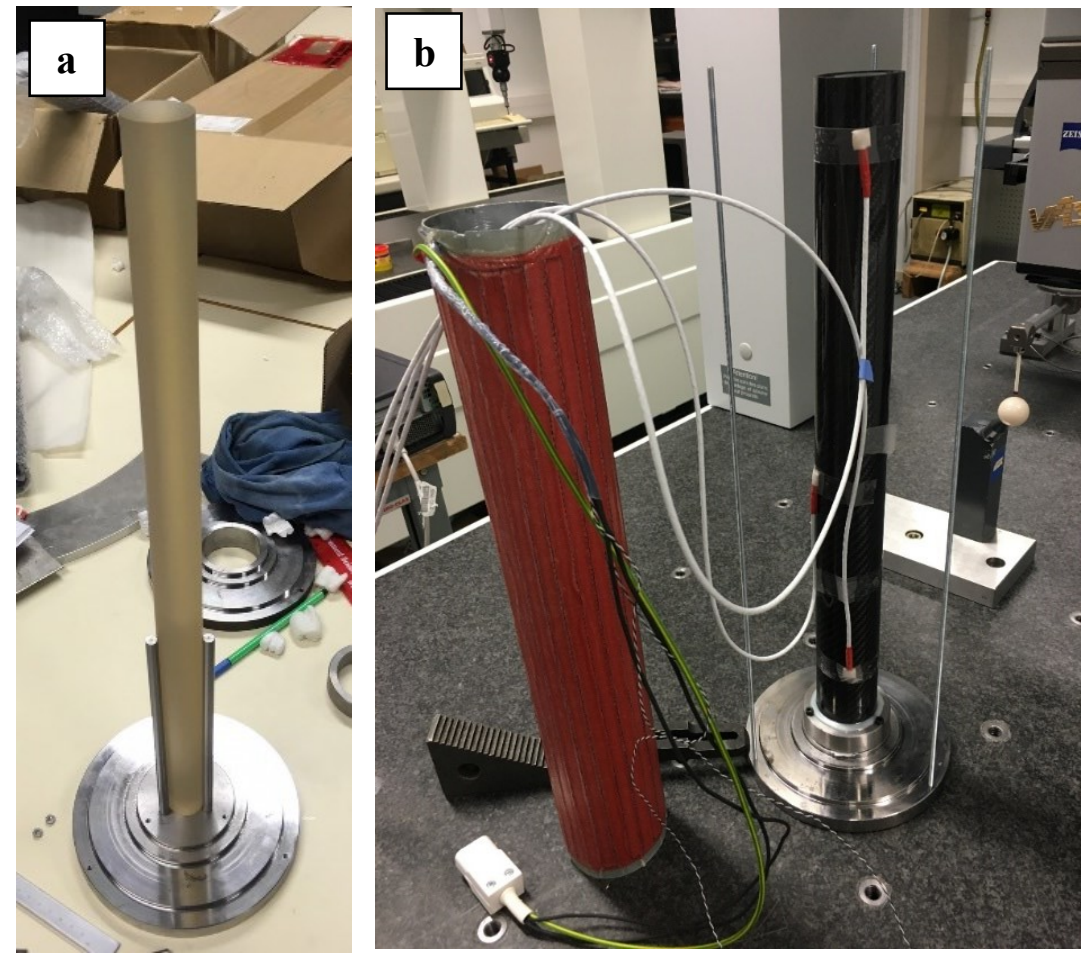

Fig. 1.a) ZERODUR® in its socket. - b) Heating tube and instrumented carbon tube.

Fig. 2 presents the schematic diagram of the assembly of the carbon tube and the ZERODUR ${ }^{\circledR}$ in the heating chamber, allowing the characterization of changes in the length of the carbon tube as a function of temperature. The glass wool insulation is held in place by 2 PVC pipe pipes of diameters $100 \mathrm{~mm}$ and $160 \mathrm{~mm}$ respectively. A shim made of thermally insulating material was installed between the heating assembly and the CMM table to prevent diffusion thermal leakage.

Fig. 3a shows the assembly before closing. The device is closed by a top plate to help hold the assembly in place, isolate the system from the outside and thus ensure quality measurements on the CMM (Fig. 3b). 


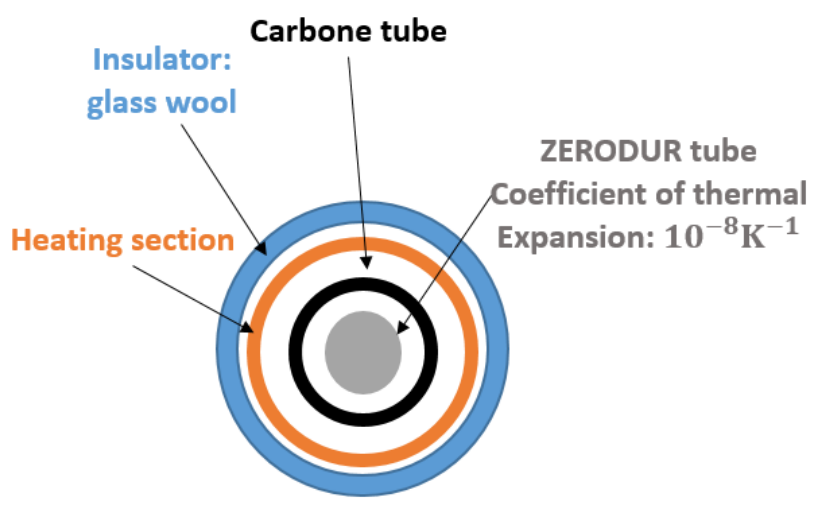

Fig. 2. Diagram of the assembly set up for the thermal characterization of carbon tube.
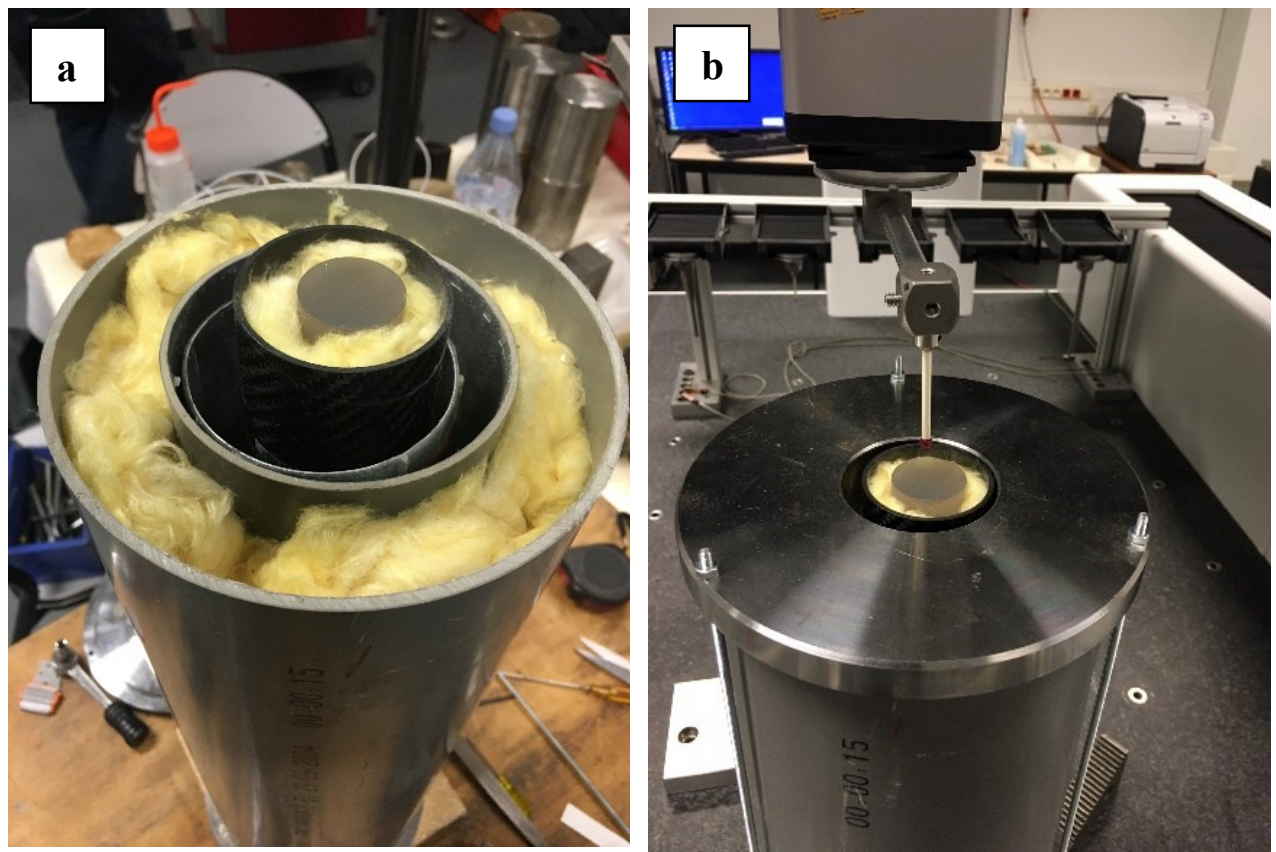

Fig. 3. a) Device before closing. - b) Device installed on the CMM.

\section{Experiments}

\subsection{Evaluation of the coefficient of thermal expansion of the carbon tube}

The assembly is gradually heated up to $30^{\circ} \mathrm{C}$ and the height variations between the upper face of the ZERODUR ${ }^{\circledR}$ and the upper plane of the carbon tube are noted and saved.

The tests were carried out 3 times at 4 different temperatures $\left(22^{\circ} \mathrm{C}, 25^{\circ} \mathrm{C}, 27^{\circ} \mathrm{C}\right.$ and 30 $\left.{ }^{\circ} \mathrm{C}\right)$.

The CTE of the carbon tube is then estimated at $1.210^{-6} \mathrm{~K}^{-1}$ with an uncertainty of $0.210^{-6}$ $\mathrm{K}^{-1}(\mathrm{k}=2)$ essentially related to thermal leakage and temperature gradients. in the tube (about $1{ }^{\circ} \mathrm{C}$ thermal gradient between the top and bottom of the tube at $30{ }^{\circ} \mathrm{C}$ for example). Measurements of height variation on CMM are repeatable to about $0.3 \mu \mathrm{m}$. 


\subsection{Evaluation of the coefficient of thermal expansion of the assembly}

The objective now is to determine the CTE associated with the assembly of the carbon tube on a structure, therefore of a carbon tube provided with two layers (one at each end). The layers are in aluminium, which is conventionally encountered on this type of structure for reasons of weight and ease of machining parts.

\subsubsection{Presentation of the tube and its layers}

The diagram of the tube with its layers is shown in Fig. 4. The original carbon tubes has been cut so that the final assembly maintains the same height.

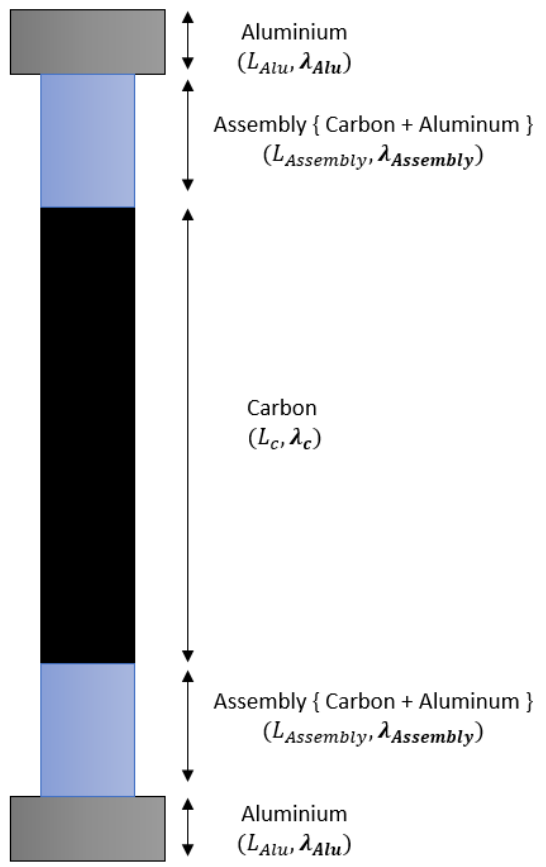

Fig. 4. Carbon tube equipped with aluminium layers at each end.

The chosen aluminium has a coefficient of thermal expansion of $\lambda_{A l u}=23.110^{-6} \mathrm{~K}^{-1}$ according to the supplier's material certificate and $\lambda_{c}$ is known from the previous experiment.

The coefficient of expansion of the assembly between the carbon tube and the aluminium alloy is noted $\lambda_{\text {Assembly }}$. The lengths of the components are known. Let it be mentioned that The use of other materials such as titanium to replace commonly used aluminum can also improve stability.

\subsubsection{Calculations and tests for determining the coefficient of expansion $\lambda_{\text {Assembly }}$}

Experimentally, the variation of the length of the complete tube $D_{\text {tube }}=1,5 \mu \mathrm{mK}^{-1}$, consisting of sections having different thermal behaviors is measured via the CMM in relative measurement with respect to the ZERODUR ${ }^{\circledR}$, as previously. The variation in length of the assembly as a function of the temperature is $D_{\text {tube }}=1.5 \mu \mathrm{mK}^{-1}$.

Thus the variation of length of the tube is governed by the relation: 


$$
2 \lambda_{\text {Alu }} \cdot L_{\text {Alu }}+2 \lambda_{\text {Assembly }} \cdot L_{\text {Assembly }}+\lambda_{c} \cdot L_{c}=D_{\text {tube }}=1,5 \mu \mathrm{mK}^{-1}
$$

Finally :

$$
\lambda_{\text {Assembly }}=\frac{1.5-\lambda_{c} \cdot L_{c}-2 \lambda_{\text {Alu }} \cdot L_{A l u}}{2 L_{\text {Assembly }}}=1.310^{-6} \mathrm{~K}^{-1}
$$

\section{Conclusion and perspective}

The studies carried out make it possible to show that the failure to take into account the coefficients of expansion of the assemblies can have heavy consequences on the variations of length. If we consider a segmented Ball Bar that would be equipped with this type of inserts, the error on the length caused by a $35 \mathrm{~mm}$ long insert integrated into a carbon tube corresponds to an error of $0.2 \mu \mathrm{mK}^{-1}$.

If we consider a Ball Bar consisting of 5 sections so 10 inserts, the error on the length is then 2 microns per degree. The error is therefore far from negligible for devices installed in uncontrolled environments. Unfortunately, since calibrations are done in an air-conditioned laboratory, this type of problem is never observed and can cause large errors in applications in an uncontrolled environment.

The good knowledge of the materials used and the consequence of their assembly is an important work that must be carried out in order to improve the corrections of metrology devices for the needs of the factory of the future.

\section{References}

1. R. Shubham, D. Rahul, J. Shivang and G.D. Acharya, "Experimental evaluation of Coefficient of Thermal Expansion of Carbon Fiber Reinforced Polymer tube," IJARED 4, (2017).

2. D. Chengzhi, L. Kai, J. Yuxi, A. Dwayne, and Z. Dongsheng, "Evaluation of thermal expansion coefficient of carbon fiber reinforced composites using electronic speckle interferometry," Opt Express 26, pp. 531-543, (2018).

3. K. Shirasu, G. Yamamoto, I. Tamaki, T. Ogasawara, Y. Shimamura, Y. Inoue and T. Hashida, "Negative axial thermal expansion coefficient of carbon nanotubes:," Carbon, 95, (2015).

4. R. Jedamzik, T. Johansson and T. Westerhoff, "Modeling of the thermal expansion behavior of ZERODUR (R) at arbitrary temperature profiles," in Proceedings of SPIE, (2010). 5.

5. [Online]. Available: https://www.schott.com/english/index.html. 